\title{
Hyperattenuated Intracerebral Lesions after Mechanical Recanalization in Acute Stroke
}

\author{
N. Lummel, G. Schulte-Altedorneburg, C. Bernau, T. Pfefferkorn, M. Patzig, H. Janssen, C. Opherk, H. Brückmann, and J. Linn
}

\begin{abstract}
BACKGROUND AND PURPOSE: Following mechanical recanalization of an acute intracranial vessel occlusion, hyperattenuated lesions are frequently found on postinterventional cranial CT. They represent either blood or-more frequently-enhancement of contrast agent. Here, we aimed to evaluate the prognostic value of these hyperattenuated intracerebral lesions.
\end{abstract}

MATERIALS AND METHODS: One hundred one consecutive patients with acute stroke in the anterior circulation who underwent mechanical recanalization were included. Risk factors for hyperattenuated intracerebral lesions were assessed, and lesion volume was compared with the volume of final infarction. Clinical outcome and relative risk of secondary hemorrhage were determined in patients with and without any hyperattenuated lesions and compared.

RESULTS: The frequency of hyperattenuated lesions was $84.2 \%$. Risk factors for hyperattenuated lesions were female sex, higher NIHSS score on admission, and higher amount of contrast agent applied. On follow-up, 3 patients showed no infarction; 53 patients, an ischemic infarction; and 45 patients, a hemorrhagic infarction. In all except 1 case, final volume of infarction (median $=92.4 \mathrm{~mL}$ ) exceeded the volume of hyperattenuated intracerebral lesions (median $=5.6 \mathrm{~mL}$ ). Patients with hyperattenuated lesions were at a 4 times higher relative risk for hemorrhagic transformation but had no significantly worse clinical outcome.

CONCLUSIONS: Our data show that the extent of postinterventional hyperattenuated intracerebral lesions underestimates the volume of final infarction. Although hyperattenuated lesions indicate a higher risk of secondary hemorrhagic transformation, their presence seems not to be of any prognostic value regarding clinical outcome.

ABBREVIATIONS: $\mathrm{CCT}=$ cranial $\mathrm{CT}$; ECASS = European Cooperative Acute Stroke Study; $\mathrm{HI}=$ hemorrhagic infarction; $\mathrm{IQR}=$ interquartile range; $\mathrm{PH}=$ parenchymal hemorrhage

$\mathbf{H}$ yperattenuated intracerebral lesions are a frequent finding on postinterventional cranial CT following intra-arterial reperfusion therapy in acute ischemic stroke. ${ }^{1-5}$ These hyperattenuated lesions have been documented and described since the 1990s. It has been suggested that hyperattenuated lesions persisting longer than 24 hours correlate with hemorrhagic lesions, whereas hyperattenuated intracerebral lesions that are no longer discernible on the 24-hour follow-up examination correspond to

Received March 5, 2013; accepted after revision April 30.

From the Departments of Neuroradiology (N.L., M.P., H.J., H.B., J.L.) and Neurology (T.P., C.O.), and Institute for Stroke and Dementia Research (C.O.), Klinikum Grosshadern, Ludwig-Maximilian-University, Munich, Germany; Institute of Radiology, Neuroradiology, and Nuclear Medicine (G.S.-A.), Klinikum Harlaching, Munich, Ger many; and Institute for Medical Information Sciences, Biometry, and Epidemiology (C.B.), Ludwig-Maximilian-University, Munich, Germany.

Please address correspondence to Nina Lummel, MD, Department of Neuroradiology, Ludwig-Maximilian-University Marchioninistr 15, 81377 Munich, Germany;

e-mail: nina.lummel@med.uni-muenchen.de

三 Indicates article with supplemental on-line appendix and tables.

http://dx.doi.org/10.3174/ajnr.A3656 contrast enhancement. ${ }^{6,7}$ The presence of hyperattenuated intracerebral lesions was considered a risk factor for secondary hemorrhage accompanied by deterioration of neurologic symptoms. ${ }^{3-5}$ However, hyperattenuated lesions do not always indicate hemorrhage, and rapid clearance of the contrast medium on postinterventional cranial CT (CCT) has been reported to be a good prognostic sign. ${ }^{1,6}$ A recent small study found no increased risk of symptomatic hemorrhage or negative clinical prognosis in patients with hyperattenuated intracerebral lesions compared with those without. ${ }^{8}$ On the other hand, Costalat et $\mathrm{al}^{9}$ recently reported that hyperattenuated intracerebral lesions on postinterventional CCT were significantly associated with poorer clinical outcome. To our knowledge, larger studies on the prognostic value of hyperattenuated intracerebral lesions are lacking to date.

Thus, the aim of this study was to further evaluate the prevalence of hyperattenuated lesions following mechanical recanalization of acute vessel occlusions in the anterior circulation, to identify risk factors for their occurrence, and to assess their prognostic value. 

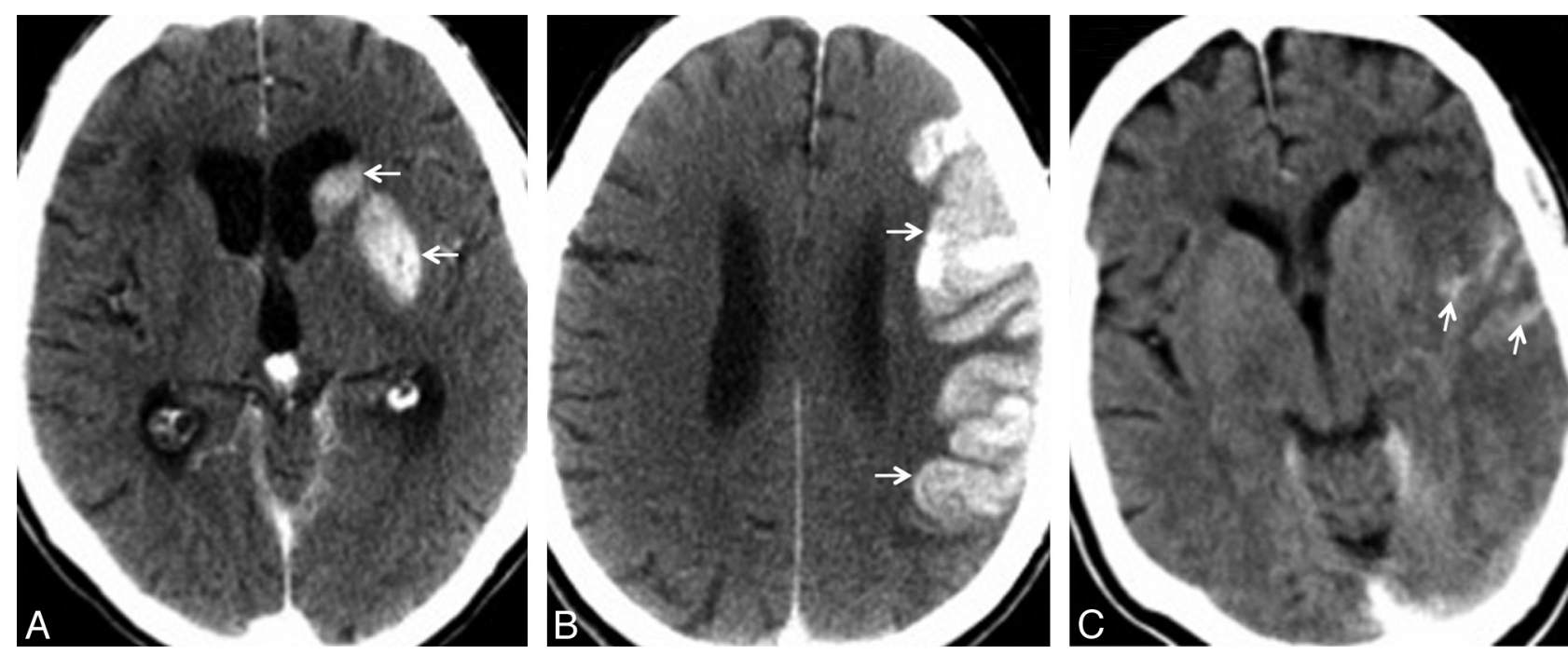

FIG 1. Postinterventional CCT of 3 different patients with hyperattenuated lesions in the basal ganglia ( $A$, arrows), cortex ( $B$, arrows), and cortical sulci ( $C$, arrows).

\section{MATERIALS AND METHODS}

The study was approved by our institutional review committee, and subjects or relatives gave their informed consent.

\section{Study Population}

All patients with acute stroke who were treated by endovascular mechanical recanalization between February 2007 and February 2012 were identified retrospectively from our in-hospital data base of angiographic records. Details on patient selection for endovascular stroke treatment are given in the On-line Appendix.

Patients were included in the analysis if they fulfilled the following additional inclusion criteria:

- Availability of an initial, complete CT stroke protocol including CCT, CTA, and CTP of evaluable diagnostic quality

- Occlusion of a large intracranial artery of the anterior circulation (common trunk of the MCA or intracranial segment of the ICA)

- Endovascular mechanical recanalization by retriever, suction devices, and/or temporary stent performed

- Availability of a postinterventional CCT performed immediately after endovascular treatment to assess the prevalence of hyperattenuated lesions

- Existence of follow-up CT performed $24 \pm 12$ hours after treatment (follow-up 1), to distinguish contrast enhancement versus hemorrhagic lesions as detailed below

- Availability of follow-up imaging (MR imaging if available, otherwise CCT) performed $>48$ hours after treatment (follow-up 2), to determine the final volume of the infarction and to assess secondary hemorrhage

- Periprocedural and clinical parameters were derived from the peri-interventional protocol and the clinical records as detailed in the On-line Appendix. The patient's clinical state on admission was rated by using the NIHSS, and clinical outcome was measured in terms of the mRS 90 days after the event. An mRS of $0-3$ was defined as good, and an mRS of 4 or 5 and death were considered poor outcomes. ${ }^{10}$

\section{Image Analysis}

Image analyses were performed by 2 experienced neuroradiologists in consensus. For details on imaging parameters and image analysis see the On-line Appendix. Briefly, pretherapeutic noncontrast CCT and CT perfusion were analyzed for the presence of early signs of ischemia, according to the ASPECTS; and CT angiography was analyzed for the presence and site of the intracranial vessel occlusion. The presence and topographic distribution of hyperattenuated lesions were determined on the postinterventional CCTs (Fig 1). Hyperattenuated lesions that were no longer discernible on the 24-hour follow-up examination were defined as contrast enhancement; hyperattenuated lesions that persisted on follow-up 1 were considered hemorrhagic lesions. ${ }^{6}$

Final stroke lesion volume and type were determined on follow-up 2 scans on the basis of the ASPECTS, also used for MR imaging, ${ }^{11}$ and by manual volume measurements as described in the On-line Appendix.

\section{Statistical Analysis}

Univariate comparisons between the 2 groups were performed by using the Fisher exact test (categoric variables) and the Mann-Whitney $U$ test (continuous variables). For univariate comparison of continuous variables among $>2$ groups, the Kruskal-Wallis rank sum test was applied. To evaluate risk factors for the incidence of hyperattenuated lesions, we performed logistic regression analyses. The Fisher exact test, ORs, and $95 \%$ CIs were determined to evaluate the association of hyperattenuated lesions with the risk of hemorrhagic transformation within the first 3 weeks. Logistic regression analyses with Akaike information criterion-based stepwise variable selection were used to evaluate whether different clinical and/or imaging factors were associated with clinical outcome. The clinical and periprocedural parameters that were tested are detailed in the On-line Appendix. We chose a value of $P=.05$ as a level of statistical significance. 


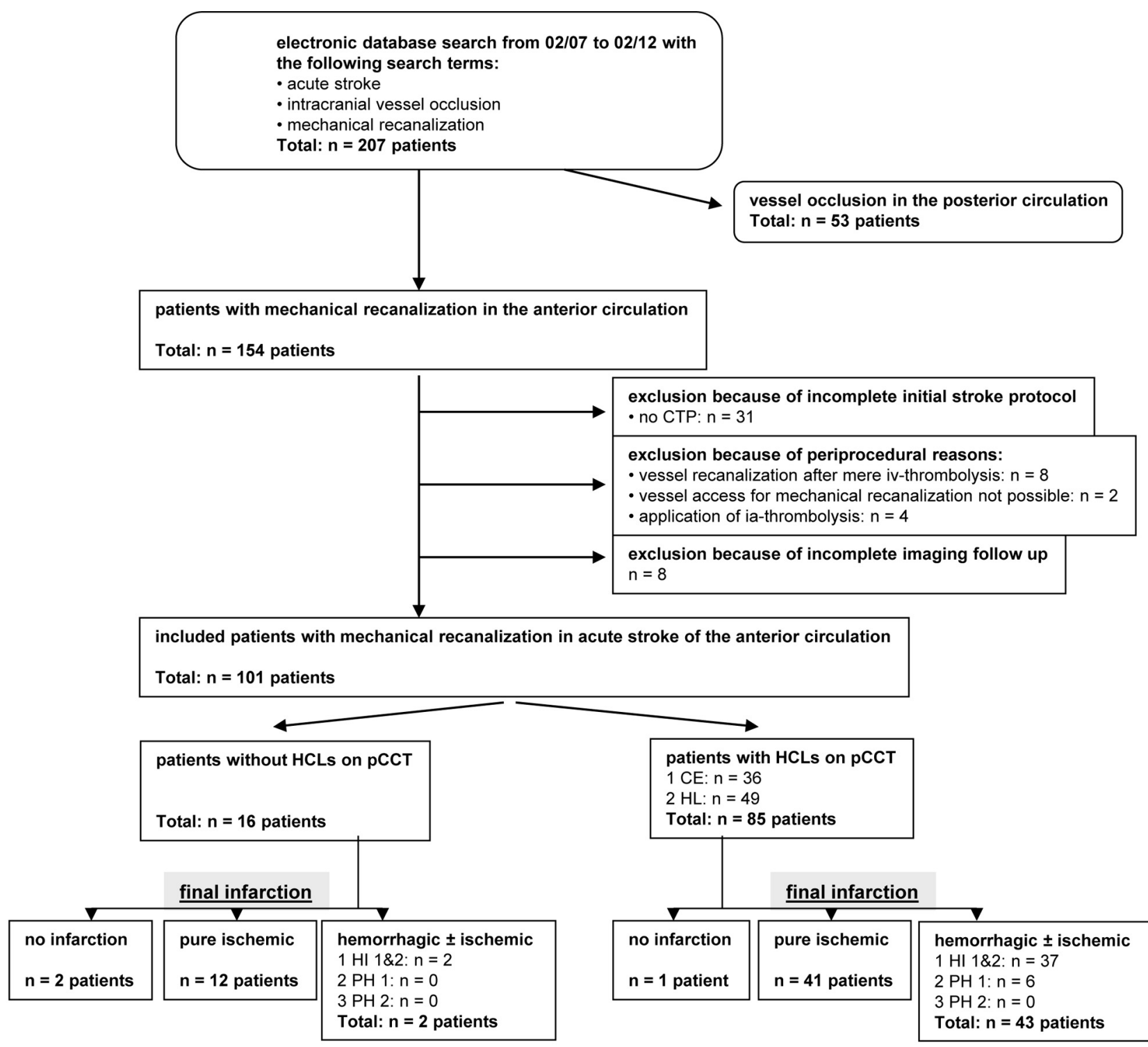

FIG 2. Flow diagram of patient identification and imaging findings. HCLs indicate hyperattenuating intracerebral lesions; $\mathrm{pCCT}$, postinterventional cranial CT; CE, contrast enhancement; $\mathrm{HT}$, hemorrhagic transformation.

\section{RESULTS}

One hundred one patients (63 women; median age, 71 years; range, 30-98 years) matched the inclusion criteria and were enrolled in this study (Fig 2). Demographic and clinical patient data, pretherapeutic imaging findings, and treatment details are summarized in Table 1 and On-line Tables 1 and 2.

The overall prevalence of hyperattenuated lesions on postinterventional CCT was $84.2 \%$. Median mean attenuation was 52 $\mathrm{HU}$ (interquartile range [IQR], $15 \mathrm{HU}$ ) and median maximum attenuation was $94 \mathrm{HU}(\mathrm{IQR}=61 \mathrm{HU})$ (Table 2). The basal ganglia were involved in $72.3 \%$. In 36 cases (35.6\%), hyperattenuated intracerebral lesions resolved on follow-up 1 , which was performed at a median interval of 17 hours after the postinterventional CCT, and were, therefore, interpreted as contrast enhancement, while hyperattenuated lesions persisted on follow-up 1 in 49 cases (48.5\%) and were thus interpreted as hemorrhagic lesions.

In univariate analysis, higher NIHSS scores on admission, larger amount of contrast agent applied, and longer duration of mechanical recanalization were significantly associated with hyperattenuated lesions (On-line Table 3). There was a tendency for hyperattenuated lesions to be more frequently observed in patients who had not received IV thrombolysis, compared with those with IV thrombolysis $(P=.06)$; however, the difference was not statistically significant. The amount of IV thrombolysis showed no significant influence on the occurrence of hyperattenuated lesions $(P=.41)$. For details on clinical, imaging, and therapy-related differences between patients with and without hyperattenuated lesions, see On-line Table 3.

The multivariate analysis primarily confirmed results of the univariate analyses. In the multivariate analysis, sex, NIHSS score on admission, and amount of contrast agent applied emerged as relevant influential factors on the occurrence of hyperattenuated lesions, whereby female sex (OR, 5.13; 95\% CI, 1.34-19.52), higher NIHSS value (OR, 1.22; 95\% CI, 1.02-1.46), and a higher 
Table 1: Demographic and clinical characteristics of the study population ( $N=101$ patients)

\begin{tabular}{lcccc}
\hline & No. $^{\text {a }}$ & No. (\%) & Median & IQR \\
\hline Age (yr) & 101 & & 71 & 21.5 \\
Sex & 101 & & & \\
$\quad$ Men & & $38(37.6 \%)$ & & \\
$\quad$ Women & & $63(62.4 \%)$ & & \\
NIHSS score on admission & 98 & & 15 & 6 \\
No. of CVRFs & 81 & & 1 & 1 \\
Etiology of stroke & 101 & & & \\
$\quad$ TOAST 1 & & $17(16.8 \%)$ & & \\
TOAST 2 & & $63(62.4 \%)$ & & \\
TOAST 3 & & $5(5 \%)$ & & \\
TOAST 4 & & $16(15.8 \%)$ & & \\
Clinical outcome & 98 & & $44(33.7 \%)$ & \\
Good (mRS 0-3) & & & $5(5 \%)$ & \\
$\quad$ mRS 0 & & & $5(5 \%)$ & \\
$\quad$ mRS 1 & & & $13(12.9 \%)$ & \\
$\quad$ mRS 2 & & $11(10.9 \%)$ & \\
$\quad$ mRS 3 & & & $64(63.4 \%)$ & \\
Poor (mRS 4-6) & & & $39(38.6 \%)$ & \\
$\quad$ mRS 4 & & $14(13.9 \%)$ & \\
$\quad$ mRS 5 & & $11(10.9 \%)$ & \\
mRS 6 & & &
\end{tabular}

Note:-CVRF indicates cardiovascular risk factors; Trial of Org 10172 in Acute Stroke Treatment (TOAST) 1, large-artery atherosclerosis; 2, cardioemboly; 3, dissection; 4, unknown etiology.

${ }^{a}$ No. indicates number in which the parameter was available in this retrospective study.

\begin{tabular}{|c|c|c|c|c|c|c|c|}
\hline & \multirow[b]{2}{*}{ No. } & \multicolumn{3}{|c|}{ HU Mean } & \multicolumn{3}{|c|}{ HU Maximum } \\
\hline & & Median & IQR & $P$ Value & Median & IQR & $P$ Value \\
\hline $\mathrm{HCL}$ on $\mathrm{pCCT}$ & 75 & 52 & 15 & & 94 & 61 & \\
\hline Follow-up 1 & 75 & & & & & & \\
\hline \multirow[t]{2}{*}{ CE } & 29 & 45 & 4 & \multirow{3}{*}{$<.001$} & 72 & 19.5 & \multirow[b]{2}{*}{$<.001$} \\
\hline & & & & & & & \\
\hline \multirow{4}{*}{$\begin{array}{l}\mathrm{HL} \\
\text { Final infarction } \\
\text { Ischemic }\end{array}$} & 46 & 58 & 13.5 & & 113 & 59.5 & \\
\hline & $74^{b}$ & & & & & & \\
\hline & 35 & 46 & 7 & \multirow{3}{*}{$<.001$} & 80 & 27 & \multirow{3}{*}{$<.001$} \\
\hline & & & & & & & \\
\hline Hemorrhagic & 39 & 58 & 11 & & 109 & 68 & \\
\hline
\end{tabular}

Note:- $\mathrm{HCL}$ indicates hyperattenuated intracerebral lesion; $\mathrm{CE}$, contrast enhancement; HL, hemorrhagic lesion; PCCT, postinterventional cranial CT.

${ }^{a}$ All patients with hyperattenuated intracerebral lesions in the basal ganglia and cortex; patients with pure sulcal hyperattenuating lesions $(n=10)$ are excluded in this calculation because Hounsfield unit values were not evaluated in these patients.

${ }^{\mathrm{b}}$ One case with hyperattenuated lesions on postinterventional CCT had no infarction on follow-up 2 and was therefore excluded.

amount of contrast agent (OR, 1.0; 95\% CI, 1.0-1.01) led to a higher risk of hyperattenuated lesions $(P$ overall $=.001)$.

The median time between follow-ups 1 and 2 was 4 days. MR imaging was available as follow-up 2 in 54 patients. Except for 1 patient, final volume of infarction (median, $92.4 \mathrm{~mL}$; IQR, 177.7 $\mathrm{mL}$ ) consistently exceeded the volume of hyperattenuated intracerebral lesions (median, $5.6 \mathrm{~mL}$; IQR, $16.2 \mathrm{~mL}$ ). Of the 36 patients with contrast enhancement on follow-up 1, one patient showed no infarction on follow-up 2, thirty-one patients developed pure ischemic lesions, and 4 cases demonstrated secondary hemorrhagic transformation (HI) (HI1/2 [see On-line Appendix], no parenchymal hemorrhage $[\mathrm{PH}] 1 / 2)$. Of the 49 patients with hemorrhagic lesions on follow-up 1, final lesions were pure ischemic in 10 cases and hemorrhagic in 39 cases (HI1/2, $n=33$;
$\mathrm{PH} 1, n=6$; PH2, $n=0$ ). In 2 of the hemorrhagic lesion cases with small hematomas on follow-up 2, localization of the bleeding did not accord with localization of hyperattenuated lesions on postinterventional CCT (Fig 3). Patients without any hyperattenuated lesions $(n=16)$ showed no infarction on follow-up 2 in 2 cases, a pure ischemic stroke in 12 cases, and a secondary hemorrhagic transformation in 2 cases (all HI1/2, no $\mathrm{PH} 1 / 2$ ).

In the univariate analysis, the following factors were significantly associated with poor clinical outcome: older age, higher NIHSS scores on admission, lower ASPECTS on the initial CCT, low degree of mismatch on CTP, longer time between initial CCT and start of the intervention, and unsuccessful recanalization (TICI 0,1 , or 2a) (On-line Table 4).

The multivariate analysis primarily confirmed results of the univariate analyses. In the multivariate analysis, age, NIHSS score on admission, ASPECTS on the initial CCT, degree of mismatch on CTP, and recanalization result emerged as important influential factors on the clinical outcome, whereby older age (OR, 1.07; 95\% CI, 1.02-1.12), higher NIHSS values (OR, 1.20; 95\% CI, 1.02-1.40), lower ASPECTS values (OR, 0.77; 95\% CI, $0.58-$ 1.02 ), lower degree of mismatch (OR, $0.78 ; 95 \% \mathrm{CI}, 0.61-1.00$ ), and unsuccessful recanalization (OR, 0.02; 95\% CI, 0.00-0.31) led to a higher risk of poor clinical outcome $(P$ overall $=1.5 \times$ $\left.10^{-8}\right)$.

\section{DISCUSSION}

Hyperattenuated intracerebral lesions are a common finding on CCT following mechanical recanalization in ischemic stroke. Our data show that female sex, a poor clinical condition on admission, and a high amount of contrast agent applied during mechanical thrombectomy correlate with their occurrence. Concerning their prognostic value, we found hyperattenuated lesions significantly more often associated with hemorrhagic transformation of the infarction. However, hyperattenuated lesions seem to be no predictor of poor clinical outcome.

In the 1990s, Wildenhain et $\mathrm{al}^{1}$ and Komiyama et $\mathrm{al}^{2}$ were among the first to document the occurrence of high-attenuation lesions on CT scans immediately after intra-arterial thrombolysis in acute stroke. Currently, hyperattenuated intracerebral lesions on postinterventional CCT are considered a common finding, not only following intra-arterial thrombolysis but also after mechanical thrombectomy.

Compared with previous studies, the incidence of hyperattenuated lesions on postinterventional CCT was fairly high in this study ( $84 \%$ versus $\left.25 \%-78 \%^{1,6,8,9,12,13}\right)$. However, most earlier studies $^{1,6,12,13}$ evaluated hyperattenuated lesions after IA thrombolysis, while data on hyperattenuated lesions following mechanical recanalization is sparse. In studies with a comparable patient collective, hyperattenuated intracerebral lesions on postinterventional CCT were found in $40 \%$ and $54 \%$ of cases, respectively, 8,9 though other factors like a different number of patients, different mechanical retrievers, revascularization rate, and anticoagulation therapy may additionally influence the incidence of hyperattenuated intracerebral lesions. Compared with Parrilla et al, ${ }^{8}$ the percentage of patients who received IV thrombolysis before mechanical recanalization was indeed higher in our collective $(46.7 \%$ 

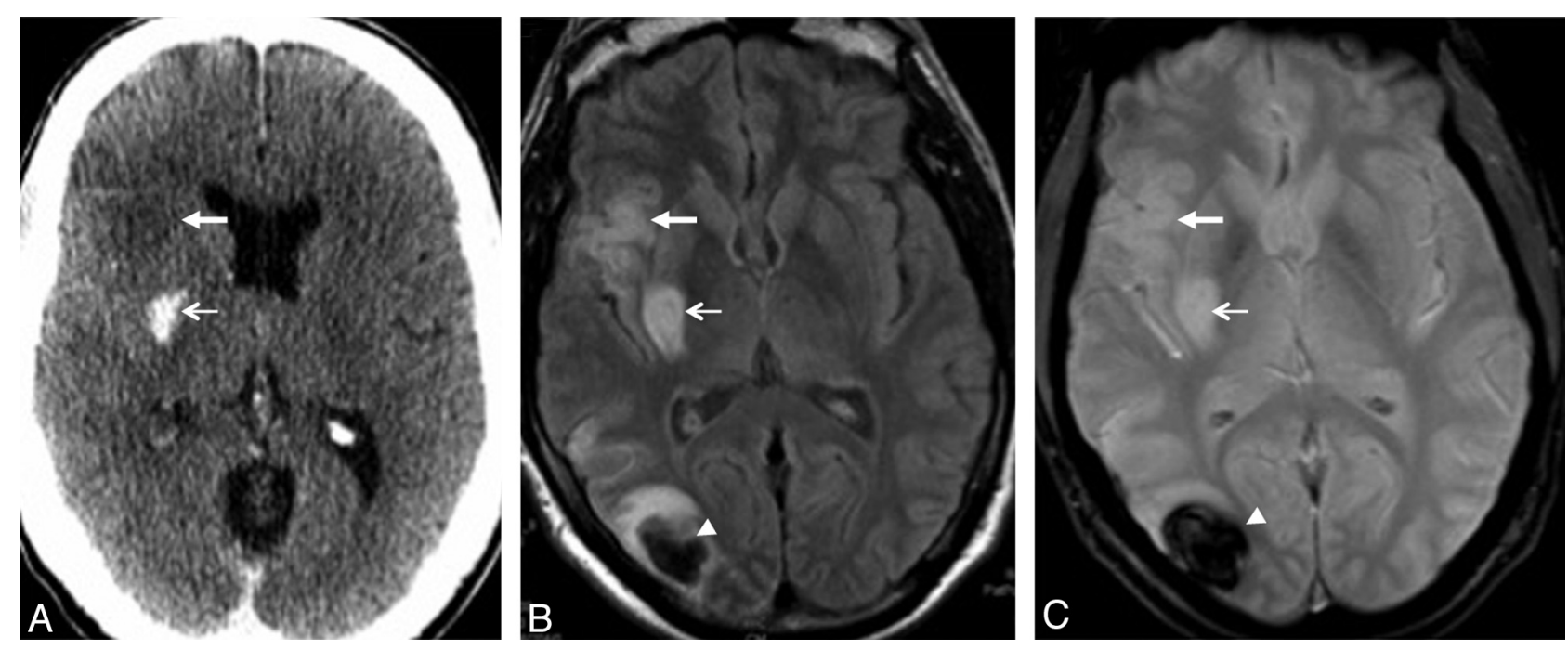

FIG 3. A 47-year-old man with acute occlusion of the right MCA and mechanical recanalization. Postinterventional CCT (A) shows a hyperattenuated intracerebral lesion in the right putamen (thin arrow) and early signs of ischemic infarction in the right anterior insula (thick arrow). MR imaging (FLAIR in $B$, and T2* in C) 2 days following the event shows pure ischemic infarction in both localizations (right putamen and anterior insula; thin and thick arrows in $B$ and $C$ ). Furthermore, MR imaging shows an intracerebral hematoma (PH1) in the right parieto-occipital lobe (arrowheads on $B$ and $C$ ), where no hyperattenuated lesion was evident on postinterventional CCT. Clinical outcome of this patient was good (mRS 1).

versus $73.3 \%$ ); however, IV lysis was not found to be a risk factor for the occurrence of hyperattenuated lesions.

Hyperattenuated intracerebral lesions do not represent a homogeneous kind of underlying parenchymal change but can reflect different pathologic changes. They correspond either to a primary or secondary parenchymal hemorrhage or to enhancement of contrast medium or a mixture of both. Unfortunately, there is no widely accepted definition for differentiating these phenomena. Referring to Yoon et al (2004), ${ }^{4}$ we distinguished 2 types of hyperattenuated lesions: 1) Hyperattenuated lesions resolving on follow-up 1 were classified as contrast enhancement, identical to those in Yoon et al; 2) hyperattenuated lesions persisting on follow-up 1 were categorized as hemorrhagic lesions in this study. We deliberately used a term different from that of Yoon et al, who classified these lesions as contrast extravasation, to stress that it is not only contrast material but also blood crossing the blood-brain barrier. Using this definition, we found contrast enhancement in $35.6 \%$ of patients and hemorrhagic lesions in $48.5 \%$ of cases.

In accordance with previous studies, we found the basal ganglia most frequently involved with hyperattenuated intracerebral lesions. ${ }^{3,4,6,13}$ This might be related to the increased vulnerability of the lenticulostriate arteries to ischemia. However, further pathomechanisms seem to be involved in the occurrence of hyperattenuated intracerebral lesions. Regarding the differentiation between contrast enhancement and hemorrhagic lesion, the most widely accepted hypothesis assumes that contrast enhancement is caused by leakage of contrast medium from vessels into the extracellular spaces because of increased permeability of the BBB. On the other hand, hemorrhagic lesions are supposed to be caused by the potential contrast toxicity, which leads to degradation of the basal lamina, associated with disruption of the BBB, and results in cellular blood element extravasation from microvessels. ${ }^{14}$ Intravascular injection of iodinated contrast media, especially superselective infusion directly into the site of acute ischemic damage, has been identified as a risk factor for disruption of the BBB. ${ }^{13-15}$
Postulates for the mechanism of contrast neurotoxicity include hyperosmolality, increased pinocytosis, and inherent chemotoxicity of the contrast agents. ${ }^{14}$ Additionally, pressure transmission from injections may contribute to BBB disruption and thus contrast extravasation. In our population, the amount of contrast agent applied during mechanical recanalization showed significant influence on the occurrence of hyperattenuated lesions on postinterventional CCT. Furthermore, in vitro studies indicate that the type of contrast media applied has an influence on the occurrence and extent of intracerebral hemorrhage. ${ }^{16}$ However, our study cannot give further evidence in this regard, because low-osmolar, nonionic contrast was used consistently. Here, additional evaluation is needed.

Furthermore, it might be anticipated that administration of thrombolysis influences the risk of BBB damage. Thrombolytic agents themselves could provoke injury to the microvascular permeability barrier, and exogenous plasminogen activators might accelerate dissolution of the BBB, microvascular basal lamina, and platelet-fibrin plugs, thereby increasing edema formation and the risk of hemorrhage. ${ }^{17}$ In this context, the type of administration of thrombolysis seems to play an important role. IA thrombolysis with direct administration of thrombolytics into the core of the infarct has been linked to a higher risk of hemorrhage compared with IV thrombolysis. ${ }^{18-20}$ In the present study, patients were included only if thrombolysis was given intravenously, and all patients with mere or additional intra-arterial thrombolysis were excluded. Hence, we found no significant association between hyperattenuated intracerebral lesions and IV thrombolysis, in accordance with former investigations. ${ }^{8}$ Neither the application of IV thrombolysis nor the amount of thrombolysis given showed a significant influence on the occurrence of hyperattenuated lesions on postinterventional CCT. Furthermore, other factors with conceivable impact on the occurrence of hyperattenuated intracerebral lesions, like cardiovascular risk factors, ASPECTS on initial CCT, type of retriever, recanalization success, 
or different time factors, showed no significant association. Only regarding the NIHSS score on admission did our data deviate from those of Parrilla et al (2012). ${ }^{8}$ In our series, patients who presented with major neurologic deficits were more prone to develop hyperattenuated lesions following recanalization.

In summary, our multivariate analysis highlights the following 3 factors as significant predictors for the occurrence of hyperattenuated lesions: 1) sex, 2) clinical condition on admission (NIHSS score), and 3) the amount of contrast agent applied during mechanical recanalization.

\section{Prognostic Value of Hyperattenuated Intracerebral Lesions}

Besides imaging characteristics and etiologic factors of hyperattenuated lesions on postinterventional CCT, their prognostic value was a major objective of this study. Some investigators suggested that hyperattenuated intracerebral lesions are highly associated with the formation of severe hemorrhagic transformation and/or parenchymal hematoma with neurologic deterioration and should thus be considered as a negative prognostic predictor for the procedure. ${ }^{3,4,13}$ In our population also, patients with hyperattenuated lesions demonstrated a clearly higher risk of developing a hemorrhagic infarction compared with patients without hyperattenuated lesions. In patients with hyperattenuated lesions on postinterventional CCT, a hematoma of $\leq 30 \%$ of the infarcted area with some mild space-occupying effect (PH1) was evident in $5.9 \%(n=6)$ of patients, in whom hemorrhage did not occur in the same localization as hyperattenuated lesions in 2 cases. Despite the increased risk for hemorrhagic transformation in patients with hyperattenuated lesions on postinterventional CCT, we, as well as Parrilla et al (2012), ${ }^{8}$ found no statistically significant influence of hyperattenuated intracerebral lesions on the clinical outcome. Accordingly, the post hoc analysis of the data of the European Cooperative Acute Stroke Study (ECASS) II trial identified only intracerebral hematomas in $>30 \%$ of the infarcted area with significant space-occupying effect (PH2) but not hemorrhagic transformation (HI1/2) or PH1 to be significantly associated with clinical deterioration and impaired prognosis. ${ }^{21}$ Even when we considered patients with contrast enhancement separately from patients with hemorrhagic lesions, there was no significant difference regarding clinical outcome in our collective, contrary to Yoon et al (2004), ${ }^{4}$ who suggested that extravasation (like our hemorrhagic lesion) portended poorer outcome than did enhancement.

Generally, occlusion of a proximal intracranial vessel is a serious disease, and our patient collective was severely clinically affected, with a high risk of persisting neurologic deficits (total median NIHSS score on admission $=15[\mathrm{IQR}=6]$, total median mRS 90 days after the event $=4[\mathrm{IQR}=2]$ ). For this reason $\mathrm{mRS}=3$ was still considered a good outcome when dichotomizing the scale. ${ }^{10}$

Among factors for good clinical outcome, time from onset of symptoms to recanalization is considered one of the key factors, with an increasing time span to recanalization decreasing the chances of success for the rescue of tissue at risk. ${ }^{22}$ In our investigation, various time factors were assessed but, as shown in previous investigations, did not show a statistically significant influ- ence on clinical outcome. ${ }^{9}$ Only a short time between initial CCT and beginning of the procedure of mechanical thrombectomy was associated with better clinical outcome in the univariate analysis, but it did not sustain multivariate testing. These results emphasize that time alone might not be as essential as suggested because other, more elusive factors, like collateralization, considerably determine the evolution of cerebral infarction. ${ }^{23}$

Our data agree with those in previous studies assigning significant influence on clinical outcome to the extent of mismatch in the initial CTP and recanalization success. ${ }^{23}$ Furthermore, we likewise found that age seems to play a critical role, implicating that younger patients with stroke might benefit even more from interventional stroke therapy. ${ }^{23}$ In our collective, a more favorable outcome was comprehensively evident in patients with lower NIHSS scores ${ }^{9}$ and higher ASPECTS on admission.

In the multivariate analysis, the following 5 factors emerged as significant predictors for clinical outcome after mechanical recanalization of an acute intracranial vessel occlusion in the anterior circulation: 1) age, 2) clinical condition an admission (NIHSS score), 3) extent of early stroke signs (ASPECTS) on the initial CCT, 4) mismatch in initial CTP, and 5) recanalization success of the cerebral artery (TICI).

\section{Limitations}

In general, the retrospective design is the major limitation of this study. Due to this approach, only the beginning of the procedure of mechanical thrombectomy, instead of the exact time of recanalization, could be validly ascertained. In addition, time factors were unknown in almost half of the patients, mainly in so-called "wake-up-strokes" or in single patients who were transferred from other hospitals where IV thrombolysis was applied without documentation of time. Another drawback of the current study is the image-based estimation of hemorrhagic transformation without histopathologic correlation. Further experimental and pathologic studies are needed regarding changes in the cerebral microvascular barriers in stroke and mechanical recanalization to clarify underlying mechanisms of hyperattenuated intracerebral lesions, particularly with respect to the differentiation between contrast enhancement and hemorrhagic lesions. Potentially, by using dual-energy CT, accurately differentiating between intracranial hemorrhage and iodinated contrast medium staining might be feasible, ${ }^{24}$ but currently this technology is not generally available. Furthermore, the lack of detailed information about prior and further anticoagulation therapy is another limitation of this study. An influence of this medication on the occurrence of hyperattenuated lesions and/or hemorrhage might be conceivable, but it was not an objective of this study. Further prospective work is needed regarding this issue.

\section{CONCLUSIONS}

In summary, our data show that hyperattenuated intracerebral lesions frequently occur on CCT following mechanical thrombectomy in acute stroke of the anterior circulation. The extent of these lesions underestimates the volume of final infarction; hence, it is of limited predictive value. In our series, hyperattenuated lesions were associated with a higher risk of hemorrhagic transformation, which might be consequential for the postinterven- 
tional drug management, and need to be evaluated circumstantially in large, prospective studies. However, hyperattenuated lesions cannot be used to forecast negative clinical outcomes.

Disclosures: Christian Opherk-UNRELATED: Board Membership: Boehringer-Ingelheim, Bristol-Myers-Squibb, Payment for Lectures (including service on Speakers Bureaus): Boehringer-Ingelheim, Bristol-Myers-Squibb, Bayer, Merck \& Co.

\section{REFERENCES}

1. Wildenhain SL, Jungreis CA, Barr J, et al. CT after intracranial intraarterial thrombolysis for acute stroke. AJNR Am J Neuroradiol 1994;15:487-92

2. Komiyama M, Nishijima Y, Nishio A, et al. Extravasation of contrast medium from the lenticulostriate artery following local intracarotid fibrinolysis. Surg Neurol 1993;39:315-19

3. Nakano S, Iseda T, Kawano H, et al. Parenchymal hyperdensity on computed tomography after intra-arterial reperfusion therapy for acute middle cerebral artery occlusion: incidence and clinical significance. Stroke 2001;32:2042-48

4. Yoon W, Seo JJ, Kim JK, et al. Contrast enhancement and contrast extravasation on computed tomography after intra-arterial thrombolysis in patients with acute ischemic stroke. Stroke 2004; 35:876-81

5. Jang YM, Lee DH, Kim HS, et al. The fate of high-density lesions on the non-contrast CT obtained immediately after intra-arterial thrombolysis in ischemic stroke patients. Korean J Radiol 2006;7:221-28

6. Mericle RA, Lopes DK, Fronckowiak MD, et al. A grading scale to predict outcomes after intra-arterial thrombolysis for stroke complicated by contrast extravasation. Neurosurgery 2000:46:1307-14, discussion 1314-15

7. Yoon W, Seo JJ, Kang HK. Bilateral paramedian thalamic contrast enhancement on CT after intra-arterial thrombolysis. Korean $\mathrm{Ja}$ diol 2005;6:41-43

8. Parrilla G, Garcia-Villalba B, Espinosa de Rueda M, et al. Hemorrhage/contrast staining areas after mechanical intra-arterial thrombectomy in acute ischemic stroke: imaging findings and clinical significance. AJNR Am J Neuroradiol 2012;33:1791-96

9. Costalat V, Lobotesis $\mathrm{K}, \mathrm{Machi} \mathrm{P}$, et al. Prognostic factors related to clinical outcome following thrombectomy in ischemic stroke (RECOST Study): $\mathbf{5 0}$ patients prospective study. Eur J Radiol 2012; 81:4075-82

10. Rittenberger JC, Raina K, Holm MB, et al. Association between Cerebral Performance Category, Modified Rankin Scale, and discharge disposition after cardiac arrest. Resuscitation 2011; 82:1036-40

11. Singer OC, Kurre W, Humpich MC, et al. Risk assessment of symp- tomatic intracerebral hemorrhage after thrombolysis using DWIASPECTS. Stroke 2009;40:2743-48

12. Urbach H, Bendszus M, Brechtelsbauer D, et al. Extravasation of contrast medium in local intra-arterial fibrinolysis of the carotid territory [In German]. Nervenarzt 1998;69:490-94

13. Yokogami K, Nakano S, Ohta H, et al. Prediction of hemorrhagic complications after thrombolytic therapy for middle cerebral artery occlusion: value of pre- and post-therapeutic computed tomographic findings and angiographic occlusive site. Neurosurgery 1996;39:1102-07

14. Khatri R, Khatri P, Khoury J, et al. Microcatheter contrast injections during intra-arterial thrombolysis increase intracranial hemorrhage risk. J Neurointerv Surg 2010;2:115-19

15. Khatri P, Broderick JP, Khoury JC, et al. Microcatheter contrast injections during intra-arterial thrombolysis may increase intracranial hemorrhage risk. Stroke 2008;39:3283-87

16. Kurosawa $\mathrm{Y}, \mathrm{Lu} \mathrm{A}$, Kjatri $\mathrm{P}$, et al. Intra-arterial iodinated radiographic contrast material injection administration in a rat middle cerebral artery occlusion and reperfusion model: possible effects on intracerebral hemorrhage. Stroke 2010;41:1013-17

17. Rudd MA, Johnstone MT, Rabbani LE, et al. Thrombolytic therapy causes an increase in vascular permeability that is reversed by 1-deamino-8-D-vasopressin. Circulation 1991;84:2568-73

18. Wahlgren N, Ahmed N, Dávalos A, et al. Thrombolysis with alteplase for acute ischaemic stroke in the Safe Implementation of Thrombolysis in Stroke-Monitoring Study (SITS-MOST): an observational study. Lancet 2007;369:275-82

19. Tissue plasminogen activator for acute ischemic stroke: the National Institute of Neurological Disorders and Stroke rt-PA Stroke Study Group. N Engl J Med 1995;333:1581-87

20. Nichols C, Khatri P, Tomsick T, et al. Advantages of a combined approach to recanalization therapy. Stroke 2008;39:e71, author reply e72

21. Berger C, Fiorelli M, Steiner T, et al. Hemorrhagic transformation of ischemic brain tissue-asymptomatic or symptomatic. Stroke 2001;32:1330-35

22. Jansen $\mathrm{O}$, Schellinger $\mathrm{P}$, Fiebach $\mathrm{J}$, et al. Early recanalisation in acute ischaemic stroke saves tissue at risk defined by MRI. Lancet 1999;353:2036-37

23. Hesselmann V, Niederstadt T, Dziewas R, et al. Reperfusion by combined thrombolysis and mechanical thrombectomy in acute stroke: effect of collateralization, mismatch, and time to and grade of recanalization on clinical and tissue outcome. AJNR Am J Neuroradiol 2012;33:336-42

24. Phan CM, Yoo AJ, Hirsch JA, et al. Differentiation of hemorrhage from iodinated contrast in different intracranial compartments using dual-energy head CT. AJNR Am J Neuroradiol 2012;33:1088-94 